\section{JTI}

JOURNAL OF

TRAUMA AND INJURY

Received: June 4, 2020

Revised: August 24, 2020

Accepted: August 31, 2020

\section{Correspondence to}

Seung Hwan Lee, M.D.

Department of Trauma Surgery, Gachon University Gil Medical Center, 21 Namdong-daero 774beon-gil, Namdong-gu, Incheon 21565, Korea

Tel: $+82-32-460-3010$

Fax: +82-32-460-2372

E-mail: surgeonrumi@gmail.com

\title{
The Effectiveness of Simulation Train- ing in an Advanced Trauma Life Support Program for General Surgery Residents: A Pilot Study
}

\author{
Myoung Jun Kim, M.D. ${ }^{1}$, Jae Gil Lee, M.D., Ph.D. ${ }^{2}$, Seung Hwan Lee, M.D. ${ }^{3}$ \\ ${ }^{1}$ Department of Surgery, Wonju Severance Christian Hospital, Yonsei University Wonju \\ College of Medicine, Wonju, Korea \\ ${ }^{2}$ Department of Surgery, Yonsei University College of Medicine, Seoul, Korea \\ ${ }^{3}$ Department of Trauma Surgery, Gachon University Gil Medical Center, Incheon, Korea
}

Purpose: Although the Advanced Trauma Life Support (ATLS) course is now taught internationally, it has not been implemented in Korea. In recent years, interest has increased in simulation as a teaching tool in the ATLS course. We therefore hypothesized that simulation training would be a useful adjunct to the ATLS course.

Methods: We designed a 1-day curriculum that included skill development workstations, expert lectures, trauma patient simulations, and group discussion for general surgery residents. We conducted a survey to evaluate participants' level of understanding of the initial evaluation and treatment of trauma patients, their degree of knowledge and technical improvement, their satisfaction with the learning goals, and their overall satisfaction with the curriculum. We then analyzed the effects before and after the training. Results: Nine residents attended this course. None of the residents initially reported that they could perform a primary survey of trauma patients. The analysis revealed significant improvements after training in the questionnaire areas of "assembly of the team and preparation for resuscitation of a trauma patient" $(p=0.008)$, "performance of a primary survey for trauma patients" ( $p=0.007$ ), "resuscitative procedures for trauma patients" ( $p=0.008)$, "importance of re-evaluation" $(p=0.007)$, "identifying the pitfalls associated with the initial assessment and management" ( $p=0.007)$, and "importance of teamwork" $(p=0.007)$.

Conclusions: After the ATLS simulation training, all participants showed significant improvements in their understanding of how to manage multiple trauma patients. Therefore, ATLS simulation training for residents will help in the management of trauma patients.

Keywords: Simulation; Trauma; Education 


\section{INTRODUCTION}

In recent years, interest in the management of severe trauma patients has dramatically increased in South Korea (hereafter, Korea). The number of regional trauma centers for the professional management of severe trauma patients is increasing nationwide, and medical and administrative efforts are being made to increase the survival rate of trauma patients. Moreover, the importance of education for healthcare providers has been highlighted in order to properly treat trauma patients.

The Advanced Trauma Life Support (ATLS) program, which was first started in the United States by Dr. Styner JK in 1978, is currently conducted in 60 countries worldwide [1]. The basic concept of ATLS education is to quickly and accurately evaluate trauma patients, to judge the priority of management, to provide appropriate initial resuscitation accordingly, and to stabilize patients [2-4]. In Korea, attempts are now being made to develop or manage trauma-related educational programs based on the ATLS, but there is not yet a clear consensus.

These educational programs are conducted using various teaching methods. Simulation-based education has a number of advantages in many fields of medical education. An advantage of simulations is that they involve no direct harm to the patient and are free from legal issues and ethical concerns. In addition, trainees can gain sufficient experience by repeatedly performing the same medical skills in certain medical scenarios [5]. Simulation education programs for traumatic circumstances have been developed worldwide for doctors, nurses, and emergency medical technicians (EMTs) and have been positively regarded as educational tools [6]. In Korea, although some training programs, such as advanced cardiac life support and mechanical ventilator therapy, have been implemented using simulation methods, there is currently no official training on trauma such as ATLS. This study evaluated the effectiveness of ATLS training through simulations for general surgery residents.

\section{METHODS}

\section{Study design}

This study is a retrospective review of prospectively collected data at a single medical college in Seoul, South Korea. Simulation-based ATLS training was conducted at the clinical simulation center of a medical school on September 16, 2017. Our study analyzed the responses to pre- and post-training questionnaires regarding the simulation-based ATLS training. The ATLS curriculum used in this study was the same as that used in actual ATLS courses from the American College of Surgeons (ACS) and its Committee on Trauma (COT) [4].

Nine participants, consisting of first-, second-, and third-year general surgery residents, completed the course. The ATLS course developed by the ACS-COT was applied, since it contained the learning goals and educational content of ATLS education [4]. The instructors included two trauma faculty members and five trauma surgery fellows. Of the instructors, one faculty member and two trauma surgery fellows had completed the ATLS course developed by the ACS-COT. To ensure consistency of this simulation-based ATLS training course, seven instructors developed the curriculum and the training scenarios together. In addition, three instructors who completed the ATLS course each paired with the rest of the instructors and conducted the course. This study was approved by the Institutional Review Board of Severance Hospital, Yonsei University Health System (4-2018-0092) and all participants signed an informed consent agreement.

Our curriculum consisted of a single-day, 7-hour training, which included skill development workstations, expert lectures, trauma patient simulations, and group discussion (Fig. 1). The skill stations consisted of theoretical and hands-on courses, while the simulation sessions were divided into two groups of participants. Patient simulators for practice (SimMan $3 \mathrm{G}^{\circledast}$, Leardal, Stavanger, Norway) were prepared in each training room and the simulator was adjusted according to each of the given scenarios. The training room was equipped with a variety of equipment used for actual patient resuscitation, reflecting the situation when a trauma patient initially presents to an emergency room. This included equipment for 
emergency carts, defibrillators, various fluid and blood models, various drug models, and equipment for other procedures (Fig. 2). Before starting the scenarios for each case, the participants established roles, including the leader, assisting doctor, nurse, and EMT. After each scenario simulation, the participants and the instructor debriefed the case. At this time, each participant shared his or her opinions and discussed whether the management was appropriate in each situation and whether the evaluation and treatment of the patient, such as the primary survey, had taken place correctly. By doing so, we were able to attain our planned learning goals.

\begin{tabular}{|c|c|c|c|}
\hline & \multicolumn{3}{|c|}{ Skill station session } \\
\hline 09:00-09:15 & \multicolumn{3}{|c|}{ RSI } \\
\hline 09:15-09:25 & \multicolumn{3}{|c|}{ E-FAST } \\
\hline 09:25-09:40 & \multicolumn{3}{|c|}{ Pelvic binder, four-person logroll, internal jugular vein puncture (ultrasound-guided) } \\
\hline 09:40-09:50 & \multicolumn{3}{|c|}{ Needle thoracostomy, needle and surgical cricothyroidotomy } \\
\hline 09:50-10:00 & \multicolumn{3}{|c|}{ X-ray-based identifications of thoracic, pelvic, and spine injuries } \\
\hline & Group 1 & Group 2 & Group 3 \\
\hline 10:00-10:15 & RSI & Needle thoracostomy & E-FAST \\
\hline 10:15-10:30 & Needle and surgical cricothyroidotomy & $\begin{array}{l}\text { Internal jugular vein puncture } \\
\text { (ultrasound-guided) }\end{array}$ & Pelvic binder and four-person logroll \\
\hline 10:30-10:45 & Needle thoracostomy & E-FAST & RSI \\
\hline 10:45-11:00 & $\begin{array}{l}\text { Internal jugular vein puncture } \\
\text { (ultrasound-guided) }\end{array}$ & Pelvic binder and four-person logroll & Needle and surgical cricothyroidotomy \\
\hline 11:00-11:15 & E-FAST & RSI & Needle thoracostomy \\
\hline \multirow[t]{2}{*}{$11: 15-11: 30$} & Pelvic binder and four-person logroll & Needle and surgical cricothyroidotomy & $\begin{array}{l}\text { Internal jugular veinpuncture } \\
\text { (ultrasound-guided) }\end{array}$ \\
\hline & \multicolumn{3}{|c|}{ Scenario simulation session } \\
\hline 11:30-11:50 & Warm-up exercise & \multicolumn{2}{|c|}{ Simulation-0 } \\
\hline $11: 50-12: 10$ & Reflections & \multicolumn{2}{|c|}{ Feedback and discussion } \\
\hline 12:10-13:00 & \multicolumn{3}{|c|}{ Lunch } \\
\hline \multirow[t]{3}{*}{ 13:00-14:00 } & Lecture 1 (30 min) & \multicolumn{2}{|c|}{ Initial assessment and management: primary survey } \\
\hline & Lecture 2 (15 min) & \multicolumn{2}{|c|}{ Thoracic trauma: primary survey } \\
\hline & Lecture 3 (15 min) & \multicolumn{2}{|c|}{ Abdominal and pelvic trauma: primary survey } \\
\hline \multirow[t]{2}{*}{ 14:00-14:10 } & \multicolumn{3}{|c|}{ Break } \\
\hline & & Group 1 & Group 2 \\
\hline 14:10-14:45 & Scenario-1: hemorrhagic shock & \multicolumn{2}{|c|}{ Simulation and debriefing (20 min/15 min) } \\
\hline 14:45-15:20 & Scenario-2: obstructive shock & \multicolumn{2}{|c|}{ Simulation and debriefing (20 min/15 min) } \\
\hline 15:20-15:55 & Scenario-3: panfacial fracture with bleeding & \multicolumn{2}{|c|}{ Simulation and debriefing (20 min/15 min) } \\
\hline 15:55-16:10 & Reflections/closing remarks & \multicolumn{2}{|c|}{$\mathrm{Q} / \mathrm{A}$} \\
\hline
\end{tabular}

Fig. 1. Training curriculum. RSI: rapid sequence intubation, E-FAST: extended focused assessment with sonography in trauma. 


\section{Outcome measurements}

Before and after the training, a questionnaire was prepared. The pre-training questionnaire (see Supplementary File 1 for a list of questions) asked participants to provide their basic information, including age, gender, and ability to practice the skill. The questionnaire was used to check participants' level of understanding of the initial evaluation and treatment of trauma patients before the training. The questionnaire survey conducted after the training (see Supplementary File 2 for a list of questions) assessed participants' degree of knowledge and technical improvement after the training, satisfaction with the learning goals, and overall satisfaction with the curriculum. Participants responded with a score from 1 to 10 for each item in the questionnaire. In addition, the participants were asked to identify the most satisfactory and unsatisfactory aspects of the curriculum.

\section{Statistical analysis}

Statistical analysis was performed using SPSS for Windows version 23.0 (IBM Corp., Armonk, NY, USA). Continuous variables, such as those for pre- and post-training comparisons were analyzed using the Mann-Whitney $U$-test and expressed as medians and interquartile ranges. In all tests, $p$-values less than 0.05 were considered to indicate statistical significance.

\section{RESULTS}

\section{Baseline characteristics}

Nine residents participated in this simulation-based ATLS training (Table 1). Their mean age was 29.9 years, and they comprised six men (66.7\%) and three women (33.3\%). The participants included one $(11.1 \%)$ thirdyear, three (55.6\%) second-year, and three (33.3\%) firstyear general surgery residents.

In response to the question, "Can you perform the initial evaluation and treatment in multiple trauma patients? (Performance of a primary survey)", four (44\%) of the participants stated that they could not do so, and five $(55.6 \%)$ reported "I can do it if a professor or senior resident provides instruction”. No participants (0\%) responded that they could perform the task independently. In response to the question, "Have you ever been trained in the initial evaluation and treatment of trauma patients? (Learned primary survey before)", four $(44.4 \%)$ of the nine respondents answered "yes", while the remaining five $(55.6 \%)$ replied "no". Of the four respondents who indicated that they had received relevant training, two had been trained in medical school, while the other two had been trained during their general surgery residency.

In the pre-training questionnaire survey, all nine participants (100\%) answered "no" when asked, "Are you familiar with the concept of a primary survey for trauma
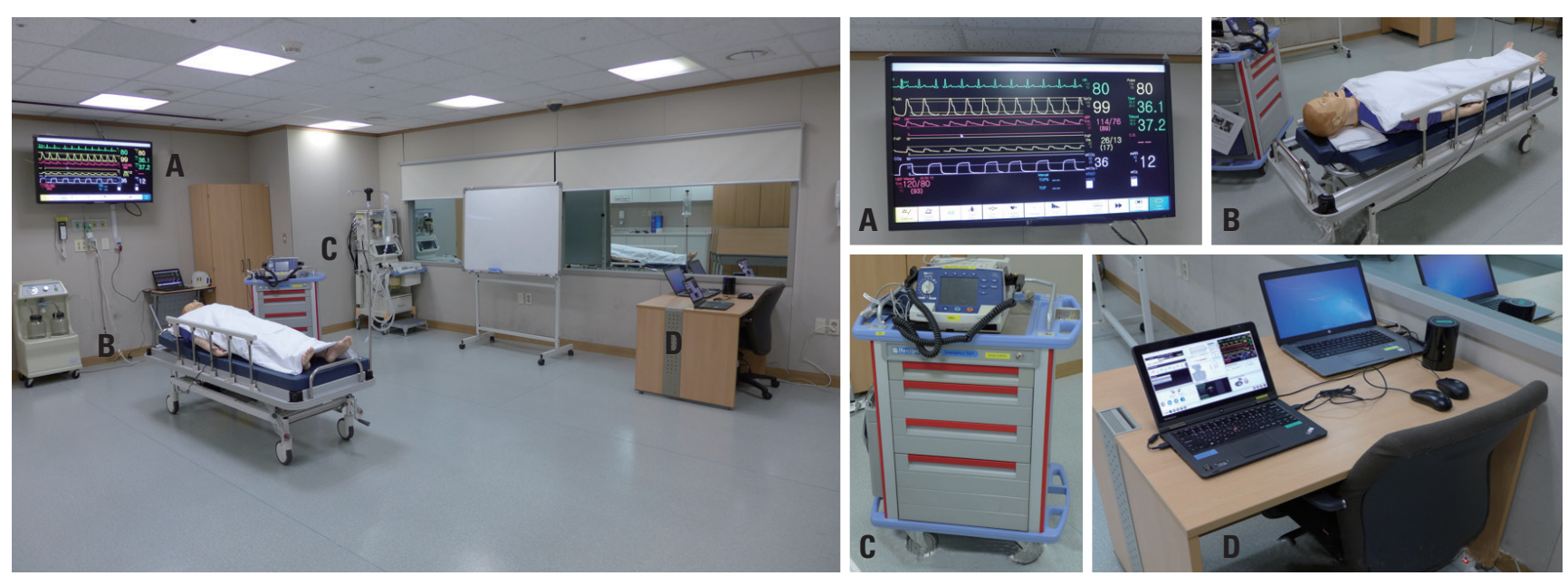

Fig. 2. Preparation for Advanced Trauma Life Support scenario simulation training. (A) Monitor, (B) patient simulator, (C) emergency cart, (D) instructor's computer. 
patients?". In response to the question "Do you know in which order the primary survey proceeds?", all nine

Table 1. Demographic characteristics of the ATLS simulation trainees

\begin{tabular}{|lc|}
\hline Variables & $p$-value \\
\hline Age (years) & $29.9(28.0-31.0)$ \\
Sex & $6(66.7)$ \\
Male & $3(33.3)$ \\
Female & \\
Year of residency & $3(33.3)$ \\
1st year & $5(55.6)$ \\
2nd year & $1(11.1)$ \\
3rd year & \\
Performance of the primary survey & $4(44.4)$ \\
Impossible & $5(55.6)$ \\
Under instruction & $0(0.0)$ \\
Independently & \\
Learned primary survey previously & $4(44.4)$ \\
Yes & $5(55.6)$ \\
No & \\
When did you learn? & $9(0.0)$ \\
Student & $2 / 4(50.0)$ \\
Resident & $2 / 4(50.0)$ \\
Basic trauma knowledge & \\
Sequence of priorities for primary survey & \\
No & \\
\hline
\end{tabular}

Values are presented as number (\%).

ATLS: Advanced Trauma Life Support. respondents answered "no" (100\%). Although almost half of the respondents had received training on trauma patient management, their actual performance skills and knowledge were insufficient.

\section{Comparison before and after training}

Based on the learning goals, we conducted a questionnaire survey to quantify participants' knowledge, skills, and attitudes related to trauma treatment before and after the training. The results are as follows (Table 2).

The items "performance of a primary survey for trauma patients" ( $p=0.007)$, "resuscitative procedures for trauma patients" ( $p=0.008$ ), and "importance of teamwork" ( $p=0.007)$ showed significant post-training increases compared to the pre-training scores. There were also significant increases in scores for the items "assembly of the team and preparation for resuscitation of a trauma patient" ( $p=0.008$ ), "significance of revaluation" ( $p=0.007$ ), and "identifying the pitfalls associated with the initial assessment and management" ( $p=0.007$ ) compared to the pre-training scores. The analysis showed that the performance of all the learning objectives was significantly higher after the training than that before the training.

\section{Curriculum satisfaction survey}

The overall satisfaction with the ATLS simulation training was high (Table 3 ). A score of $9.2 \pm 0.8$ points was given for "the usefulness of the whole curriculum", confirming the participants' satisfaction with the overall training. Among the other items, the degree of satisfaction with "the degree of professionalism of the instructors" was highest, at $9.6 \pm 0.5$ points.

The score for "appropriateness of the training time distribution for each simulation" item was $9.4 \pm 0.5$ points.

Table 2. Comparison of scores between before and after simulation training

\begin{tabular}{|c|c|c|c|}
\hline & Before & After & $p$-value \\
\hline Performance of primary survey for trauma patients & $3.0(1.0-3.5)$ & $8.0(6.0-9.0)$ & 0.007 \\
\hline Resuscitative procedures for trauma patients & $3.0(2.5-4.5)$ & $8.0(6.5-9.0)$ & 0.008 \\
\hline Importance of teamwork & $4.0(3.0-7.0)$ & $8.0(7.0-9.5)$ & 0.007 \\
\hline Assembling of team and preparation of resuscitation for trauma patient & $3.0(1.5-3.0)$ & $7.0(6.0-8.0)$ & 0.008 \\
\hline Importance of re-evaluation & $3.0(2.0-5.0)$ & $8.0(6.5-9.5)$ & 0.007 \\
\hline Identifying pitfalls associated with the initial assessment and management & $3.0(1.0-3.5)$ & $8.0(6.0-8.0)$ & 0.007 \\
\hline
\end{tabular}


"I recommend this training program as a regular course" also received a high score, with $9.4 \pm 0.9$ points. The score for "clarity of educational objectives" was $9.3 \pm 0.7$ points, while those for "appropriateness of the time allocation for each skill station", "appropriateness of the time allocation for each lecture", and "this curriculum helps me to treat trauma patients" were 9.3 $\pm 0.7,9.1 \pm 0.6$, and $9.1 \pm 0.9$ points, respectively. Participants' high level of satisfaction was confirmed by high scores not only for the general curriculum, but also for the more specific items.

\section{DISCUSSION}

One of the primary goals of medical education is to take appropriate actions in real time, especially in emergency situations. Simulation training is a useful educational tool for mastering a series of rapid procedures for patients in urgent situations, such as severe trauma patients $[5,7]$. Simulation-based education must be introduced in the area of trauma because the condition of trauma patients can suddenly worsen within a short time after injury, meaning that quick judgment and skillful procedures are necessary. The focus of the ATLS program is to establish early hemodynamic stabilization through the primary survey procedure within a short period of time after inju-

Table 3. Satisfaction in the post-education questionnaire after ATLS simulation training

\begin{tabular}{|lc|}
\hline Variables & $p$-value \\
\hline The usefulness of the entire curriculum & $9.2 \pm 0.8$ \\
\hline $\begin{array}{l}\text { Clarity of educational objectives } \\
\text { The degree of professionalism of the instructors }\end{array}$ & $9.3 \pm 0.7$ \\
$\begin{array}{l}\text { The appropriateness of the time allocation for each } \\
\text { lecture }\end{array}$ & $9.6 \pm 0.5$ \\
$\begin{array}{l}\text { The appropriateness of the time allocation for each } \\
\text { skill station }\end{array}$ & $9.3 \pm 0.7$ \\
$\begin{array}{l}\text { The appropriateness of the training time distribution } \\
\text { for each simulation }\end{array}$ & $9.4 \pm 0.5$ \\
$\begin{array}{l}\text { This curriculum helped me to treat trauma patients } \\
\text { I recommend this training program as a regular course }\end{array}$ & $9.1 \pm 0.9$ \\
\hline
\end{tabular}

Values are presented as mean \pm standard deviation.

ATLS: Advanced Trauma Life Support. ry $[4,8]$. In that sense, combining the ATLS program with simulation methods is innovative.

In recent years, a series of accidents led to growing interest in establishing a trauma treatment system in Korea, for which appropriate education is of fundamental importance. However, as seen in the pre-training questionnaire of this study, knowledge and skills on the primary survey of trauma patients and awareness of the proper approach among Korean healthcare providers remain insufficient. Most of the participants in this study were not able to independently perform the evaluation and treatment of multiple trauma patients, and most had not received education about trauma treatment. Furthermore, all participants answered that they were not familiar with the primary survey process. In foreign countries, the ATLS course involves residents, nurses, and EMTs in a variety of ways and is regularly updated $[9,10]$. Therefore, a training course such as the ATLS is necessary for constructing a trauma treatment system in Korea.

If the simulation method is introduced, the treatment strategies for patient care can be taught in a more practical way than the existing ATLS procedure. Furthermore, the simulation method has no patient safety issues [7] and is free of legal or ethical issues [11,12]. In addition, participants can acquire the necessary clinical skills to manage patients step by step and it is possible to repeat the training as many times as necessary until the trainee is able to perform the relevant skills at a competent level [13]. Another advantage of simulation-based education is that clinical situations that are rare, but inevitable, for clinicians can be practiced repeatedly for training [14]. For these reasons, simulation-based medical education is already being implemented in various fields.

In addition, education on trauma patient management through simulations has already been demonstrated to be effective in various studies. One paper reported that ATLS training of residents through a simulation resulted in a significant improvement in trauma management skills [15]. Ali et al. [16] found that the learning effects of training with actual patients and mechanical simulation models were equally good, and that the students preferred the mechanical simulation models. Knudson and Sisley [17] also showed that simulation-based training was useful for residents to develop the ability to perform skilled proce- 
dures in trauma patients, such as ultrasonography. In our study, a comparison of scores before and after the simulation-based ATLS training showed significant improvements in all items, including the performance of primary surveys, resuscitative procedures, patient revaluation, and identification of pitfalls, corresponding with previous studies.

However, there are limitations to simulation-based training, such as reduced levels of participant immersion due to the usage of a virtual patient. In addition, the anatomic demonstration may not be clearer than that provided by training using living tissue, such as cadavers. Moreover, in simulation training, the instructor artificially provides the vital signs of the patient in the simulator for practical use. Thus, if the instructor's modulation proficiency is inappropriate, the physiological and circulatory conditions may not be adequately presented as compared to animal model-based education [18].

However, a disadvantage of cadaver training is that the quality of the training can vary greatly according to the state of the cadaver; it is also difficult to reflect physiological changes that occur in certain situations. In addition, simulation-based training is relatively advantageous in comparison with live animal models because it is anatomically relevant and it can be repeated many times without incurring substantially increased costs. These benefits were also observed in the questionnaire responses from the participants. For example, participants were able to experience simulation training of various clinical scenarios by setting up simulations involving severe trauma patients, who are encountered infrequently in real-world circumstances, and were able to improve their proficiency in performing primary surveys and skilled procedures through repetition. In recent years, various hybrid model education methods using simulations have been studied, and we believe that the limitations of this method will be overcome in the future $[12,19,20]$.

This study has several limitations. First, it is a retrospective single-institution study. Second, the small sample size limits the ability to generalize the findings. The total number of participants, however, does fall within the range of sample sizes for other simulation-based trainings in the literature. Third, many subjective factors were evaluated in the questionnaire. Therefore, further refine- ment of the objectivity of the questionnaire items and the appropriateness of the evaluation criteria is necessary. In the future, we would like to prospectively assess simulation-based ATLS training in Korea through ongoing improvements and curricular development.

\section{CONCLUSION}

The simulation program was useful for ATLS training of residents. After undergoing simulation-based ATLS training, all participants showed significant improvements in their understanding and management of multiple trauma patients. In the future, we will develop a more systematic simulation education curriculum through ongoing improvement of these curricula and the development of evaluation tools. In addition, research on the potential of simulation-based training as a formal education course for residents is also needed.

\section{ACKNOWLEDGEMENTS}

The authors thank John MJ Lee for his helpful comments on this manuscript, and we thank YYC, DHC, YTJ and JYP for their valuable help and cooperation.

\section{SUPPLEMENTARY MATERIALS}

The online-only data supplement is available with this article at https://doi.org/10.20408/jti.2020.0015.

\section{REFERENCES}

1. Styner JK. The birth of Advanced Trauma Life Support (ATLS). Surgeon 2006;4:163-5.

2. van Olden GD, Meeuwis JD, Bolhuis HW, Boxma H, Goris RJ. Clinical impact of Advanced Trauma Life Support. Am J Emerg Med 2004;22:522-5.

3. Ali J, Adam R, Stedman M, Howard M, Williams J. Cognitive and attitudinal impact of the Advanced Trauma Life Support program in a developing country. J Trauma 1994;36:695-702. 
4. American College of Surgeons Committee on Trauma. ATLS ${ }^{\circledR}$ student manual. 9th ed. Chicago (IL):American College of Surgeons;2012.

5. Borggreve AS, Meijer JMR, Schreuder HWR, Ten Cate O. Simulation-based trauma education for medical students: a review of literature. Med Teach 2017;39:631-8.

6. Holcomb JB, Dumire RD, Crommett JW, Stamateris CE, Fagert MA, Cleveland JA, et al. Evaluation of trauma team performance using an advanced human patient simulator for resuscitation training. J Trauma 2002;52:1078-85; discussion 1085-6.

7. Hammond J. Simulation in critical care and trauma education and training. Curr Opin Crit Care 2004;10:325-9.

8. Girdley FM, Cohen DJ, Birnbaum ML, Bowman RM. Advanced Trauma Life Support: assessment of cognitive achievement. Mil Med 1993;158:623-7.

9. Mohammad A, Branicki F, Abu-Zidan FM. Educational and clinical impact of Advanced Trauma Life Support (ATLS) courses: a systematic review. World J Surg 2014;38:322-9.

10. Ben-Abraham R, Stein M, Shemer J, Kluger Y, Barzilay Z, Paret G. Advanced Trauma Life Support (ATLS) courses: should training be refocused towards rural physicians? Eur J Emerg Med 1999;6:111-4.

11. Scott DJ. Patient safety, competency, and the future of surgical simulation. Simul Healthc 2006;1:164-70.

12. Windsor JA. Role of simulation in surgical education and train- ing. ANZ J Surg 2009;79:127-32.

13. Cherry RA, Ali J. Current concepts in simulation-based trauma education. J Trauma 2008;65:1186-93.

14. Motola I, Devine LA, Chung HS, Sullivan JE, Issenberg SB. Simulation in healthcare education: a best evidence practical guide. AMEE Guide No. 82. Med Teach 2013;35:e1511-30.

15. Marshall RL, Smith JS, Gorman PJ, Krummel TM, Haluck RS, Cooney RN. Use of a human patient simulator in the development of resident trauma management skills. J Trauma 2001;51:17-21.

16. Ali J, Al Ahmadi K, Williams JI, Cherry RA. The standardized live patient and mechanical patient models--their roles in trauma teaching. J Trauma 2009;66:98-102.

17. Knudson MM, Sisley AC. Training residents using simulation technology: experience with ultrasound for trauma. J Trauma 2000;48:659-65.

18. Aboud ET, Krisht AF, O'Keeffe T, Nader R, Hassan M, Stevens CM, Ali F, Luchette FA. Novel simulation for training trauma surgeons. J Trauma 2011;71:1484-90.

19. Wali E, Pinto JM, Cappaert M, Lambrix M, Blood AD, Blair EA, et al. Teaching professionalism in graduate medical education: what is the role of simulation? Surgery 2016;160:552-64.

20. Simulation in trauma education: beyond ATLS. Injury 2014;45:817-8. 